\title{
Manifestaciones urológicas de la enfermedad relacionada con la inmunoglobulina G4
}

\author{
Urological manifestations of the disease related to immunoglobulin G4
}

Luis Daniel Carrillo-Córdova ${ }^{1 *}$, Carlos Alberto Carrillo-Córdova ${ }^{1}$, Johnatan Vitar-Sandoval', Jorge Jaspersen-Álvarez², Elba Luz Villena-López y Raúl Carrillo-Esper ${ }^{4}$

${ }^{1}$ Departamento de Urología, Hospital General de México Dr. Eduardo Liceaga; ${ }^{2}$ Facultad de Medicina, Universidad Nacional Autónoma de México; ${ }^{3}$ Departamento de Medicina Interna, Hospital Ángeles del Pedregal; ${ }^{4}$ División de Áreas Críticas CENIAQ, Instituto Nacional de Rehabilitación. Ciudad de México, México

\begin{abstract}
Resumen
La enfermedad relacionada con la inmunoglobulina G4 (ER-IgG4) es una enfermedad fibroinflamatoria de etiología desconocida, la cual se caracteriza por presentar lesiones en forma de tumoraciones, concentraciones séricas aumentadas de lgG4 y células plasmáticas con una infiltración importante de lgG4, junto con flebitis obliterante y fibrosis. Esta enfermedad suele tener afección multiorgánica, incluyendo el páncreas, el tracto biliar, las glándulas salivares, los tejidos periorbitarios, los riñones, los pulmones, los ganglios linfáticos y el retroperitoneo. La ER-IgG4 afecta principalmente a hombres, con un predominio de edad por los adultos jóvenes y hasta la vejez. Las manifestaciones clínicas de la ER-IgG4 dependen principalmente de los órganos afectados y de la respuesta a los esteroides. Su pronóstico aún no es del todo claro. Dentro de los órganos urogenitales afectados pueden incluirse el riñón, el retroperitoneo, el uréter, la vejiga, el uraco, el testículo/epidídimo, la región paratesticular, la próstata y la uretra.
\end{abstract}

PALABRAS CLAVE: Enfermedad por IgG4. Fibrosis retroperitoneal. Urología.

\begin{abstract}
Immunoglobulin G4 related disease (IgG4-RD) is a fibro-inflammatory disease of unknown etiology, characterized by lesions in the form of tumors, elevated serum IgG4 levels, plasma cells with significant IgG4 infiltration, accompanied by phlebitis obliterans and fibrosis. This disease usually has multiorgan disease, including pancreas, biliary tract, salivary glands, peri orbital tissues, kidneys, lungs, lymph nodes and retro peritoneum. IgG4-RD mainly affects men with a predominance of age by young adults until old age. The clinical manifestations of IgG4-RD, depend mainly on the organs affected and the response to steroids. His forecast is not yet clear. Within the affected urogenital organs can be observed kidney, retroperitoneum, ureter, bladder, urachus, testis/epididymis, paratesticular region, prostate and urethra.
\end{abstract}

KEY WORDS: IgG4-related disease. Retroperitoneal fibrosis. Urology.

\author{
Correspondencia: \\ *Luis Daniel Carrillo-Córdova \\ Cerro Teponaxtle, 109 \\ Col. Campestre Churubusco, Del. Coyoacán \\ C.P. 04200, Ciudad de México, México \\ E-mail: Carrillocor@gmail.com
}

Fecha de recepción: 08-02-2017

Fecha de aceptación: 17-08-2017

DOI://dx.doi.org/10.24875/CIRU.M18000004
Cir Cir. 2018;86:63-70

Disponible en PubMed www.cirugiaycirujanos.com 


\section{Introducción}

La enfermedad relacionada con la inmunoglobulina G4 (ER-IgG4) es una inflamación fibrótica crónica, caracterizada por la presencia de infiltración linfocítica en los tejidos, células plasmáticas positivas a $\lg \mathrm{G} 4$, desarrollo de fibrosis y en algunas ocasiones concentraciones séricas aumentadas de $\lg G 4^{1,2}$. Esta enfermedad usualmente afecta múltiples órganos. Un diagnóstico temprano es muy difícil de realizar por el curso asintomático de la enfermedad en etapas tempranas, y al presentar síntomas estos son vagos e inespecíficos. La principal manifestación suele ser secundaria a la inflamación o fibrosis de un órgano especifico, causando daño directo al mismo. También puede encontrarse de manera incidental durante el protocolo de estudio de un tumor al tomar una biopsia. En todas sus etapas, esta enfermedad suele responder bien a la terapia con esteroides ${ }^{3,4}$.

El riñón es uno de los órganos afectados con mayor frecuencia en la ER-IgG4, y las lesiones renales se engloban en un espectro llamado enfermedad renal asociada a ER-IgG44-8.Al igual que en la afección de la enfermedad a otros órganos, los pacientes son asintomáticos o tienen síntomas vagos e inespecíficos. Sin embargo, aproximadamente la mitad de estos pacientes progresarán a falla renal ${ }^{9}$.Esto muestra la necesidad de realizar un diagnóstico precoz, para iniciar el tratamiento de manera oportuna. Las lesiones renales se distribuyen desde el parénquima renal hasta la pelvis y el uréter ${ }^{3,9-16}$.

La lesión más representativa de la lesión al parénquima renal es una nefritis tubulointersticial rica en células plasmáticas, acompañada de infiltrados de células plasmáticas ricas en IgG4, y fibrosis, similar a lo observado en el retroperitoneo $0^{7,9}$.

\section{Fisiopatología}

Se cree que la enfermedad tiene un antecedente alérgico y es mediada por el sistema inmunitario, encontrando respuestas inmunitarias anormales. La adecuada respuesta a la terapia con esteroides apoya la etiología inmunitaria de esta enfermedad. Aproximadamente el $30-50 \%$ de los pacientes tienen antecedente de alergias, eosinofilia o concentraciones séricas aumentadas de $\lg \mathrm{E}^{17,18}$.

Los pacientes con ER-IgG4 presentan respuestas sobrerreguladas para Th2 (interleucina [IL] 4, IL-5, II-13 e IL-21) y células T reguladoras (IL-10 y factor de crecimiento tumoral B1). La respuesta Th2 induce una respuesta alérgica mediada por el sistema inmunitario con eosinofilia e IgE elevada ${ }^{19-22}$. Los mastocitos activados, altamente positivos para IgE, promueven la diferenciación de células $T$ vírgenes en células $T$ reguladoras. La IL-10 promueve la diferenciación de las células $B$ a células plasmáticas, con la producción subsecuente de IgG4. La IL-4 activa a los macrófagos para producir altas cantidades de IL-10. La IL-21, producida por las células Th2 y T foliculares, es importante para la formación de centros germinales y estimula la producción de IgG4 y la infiltración de células plasmáticas a los tejidos ${ }^{23,24}$. Los monocitos causan infiltración masiva de los tejidos afectados, formando folículos linfoides y produciendo el agrandamiento y la deformación del tejido afectado, lo que lleva a disfunción de los mismos; este infiltrado puede dar la apariencia de un tumor en el riñón, la pelvis renal o el uréter ${ }^{25}$.

Las células plasmáticas circulantes inducidas por antígenos tienen el fenotipo CD38+, CD27+, CD19+, CD20- y CD22- (plasmablastos de vida corta). La ER-IgG4 se asocia a una concentración elevada de plasmablastos en sangre, aun en pacientes con IgG4 sérica normal. El conteo de plasmablastos es superior que el de la concentración sérica de IgG4 como

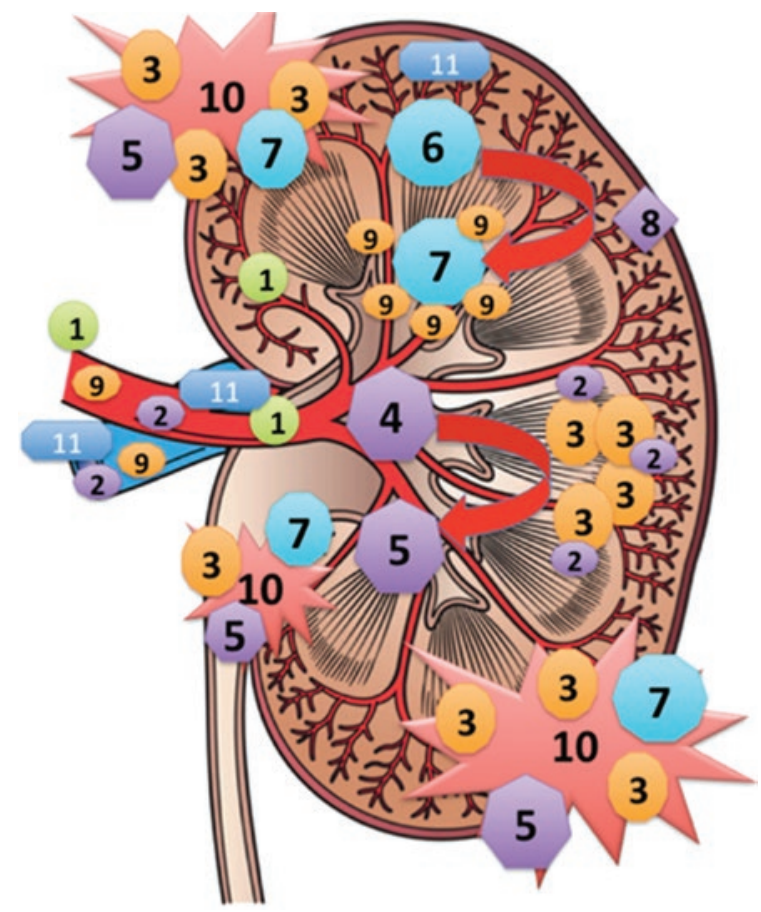

Figura 1. Fisiopatología de la enfermedad relacionada con la lgG4. Esquema que muestra las células inmunitarias y las interleucinas involucradas. 1: eosinófilos; 2: IgE; 3: mastocitos activados; 4: célula $T$ virgen; 5: célula $T$ reguladora; 6 : célula $B$; 7 : células plasmáticas; 8 : IL-10; 9: IgG4; 10: folículo linfoide; 11: plasmablasto. 
biomarcador para el diagnóstico de ER-IgG4, y es útil para el monitoreo de la enfermedad y para predecir recaídas ${ }^{26-28}$ (Fig. 1).

\section{Epidemiología}

En Japón se estima que la prevalencia es de 0.8 casos por 100,000 habitantes, y la incidencia estimada es de 0.28 a 1.08/100,000, con 336-1300 pacientes diagnosticados cada año y 6700-26,000 pacientes que desarrollaron ER-IgG4 en los últimos 20 años ${ }^{29}$. Otro estudio estimó que la prevalencia es de 6/100,000 habitantes ${ }^{30}$. Esta enfermedad afecta principalmente a personas del sexo masculino (73-80\%); con la única excepción de las manifestaciones en la cabeza y el cuello, la edad promedio de presentación va de los 50 a los 60 años ${ }^{31,32}$. Existen algunos casos en la literatura en los que esta enfermedad se ha diagnosticado en niños ${ }^{33-35}$. No hay artículos en Latinoamérica que estudien la epidemiologia de la enfermedad.

\section{Diagnóstico}

El diagnóstico de la ER-IgG4 se realiza mediante la integración de los hallazgos clínicos, los estudios de laboratorio, la histopatología típica y la radiología, como han propuesto varios autores ${ }^{36-38}$ (Fig. 2).

Un tercio de los pacientes tienen antecedente de enfermedad atópica, incluyendo asma, rinitis alérgica, pólipos nasales y dermatitis atópica; los pacientes no atópicos pueden presentar eosinofilia en sangre periférica o $\lg E$ elevada ${ }^{39}$. Los pacientes con ER-IgG4 han presentado frecuentemente alergias (hasta el $57 \%$ en una cohorte $)^{40}$.

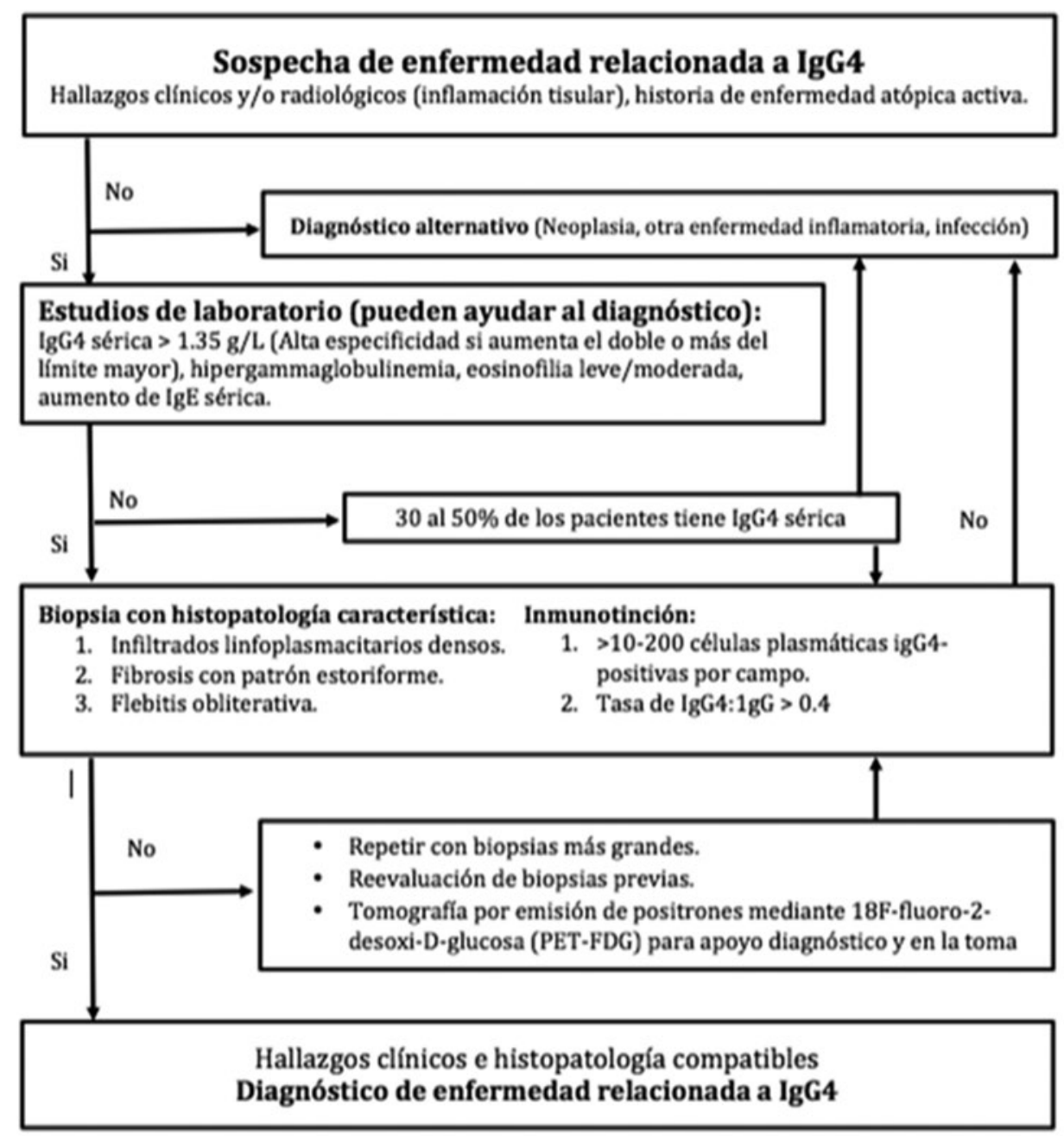

Figura 2. Algoritmo diagnóstico para la enfermedad relacionada con IgG4. Se muestra el abordaje diagnóstico propuesto por Umehara et al. ${ }^{36}$. 
Los pacientes no suelen presentar síntomas generales, como fiebre, pérdida de peso, sudor nocturno o malestar general. Se han reportado tumores malignos en el $7.4 \%$ de los pacientes con ER-IgG4. El linfoma marginal extranodal de células $B$ puede ocurrir en los anexos oculares, las glándulas salivales o la duramadre en los 5 años posteriores al diagnóstico de ER-IgG441-43.

Los estudios de laboratorio se encuentran generalmente normales, en algunas ocasiones con elevación de la proteína $C$ reactiva, una velocidad de sedimentación globular aumentada, anemia, trombocitosis y eosinofilia. La serología puede evidenciar IgE elevada, hipergammaglobulinemia policlonal, hipocomplementemia ( $21 \%$ de los casos, en especial cuando hay afectación renal por la enfermedad), presencia de anticuerpos antinucleares (16-50\%) y factor reumatoide (20\%). Una IgG4 elevada puede ayudar a establecer el diagnóstico; un valor de corte > $1.35 \mathrm{~g} / \mathrm{l}$ ha mostrado una sensibilidad del $97 \%$ y una especificidad del $79.6 \%$ para diagnosticar ER-IgG4. Sin embargo, un $30-50 \%$ de los pacientes con ERIgG4 tendrán valores séricos normales de lgG444-47.

En los estudios radiológicos pueden encontrarse pseudotumores renales, ureterales y piélicos, secundarios a la infiltración de los órganos por linfocitos y monocitos. Estas lesiones muestran atenuación de tejidos blandos y bordes bien definidos en la tomografía, y en la resonancia magnética son lesiones de isointensas a hipointensas en T2, con un reforzamiento homogéneo, lo cual refleja la celularidad y la fibrosis aumentadas; en caso de compresión extrínseca del uréter por fibrosis retroperitoneal (FRP), o intrínseca por un pseudotumor ureteral o pélvico, se podrá evidenciar hidronefrosis ${ }^{48}$.

La tomografía por emisión de positrones (PET) con 18F-fluorodesoxiglucosa permite realizar un mapeo de los sitios con inflamación al marcar áreas con actividad hipermetabólica, lo que ayuda a evaluar la extensión de la enfermedad y monitorizar su respuesta al tratamiento ${ }^{49}$.

Actualmente, el método de referencia para el diagnóstico de la ER-IgG4 es la identificación de la histología característica y la inmunohistoquímica, la cual es la misma sin importar el órgano que esté afectado. En etapas tardías de la enfermedad, lo que predomina es la fibrosis, haciendo que el diagnóstico sea más difícil ${ }^{50}$.

Las tres características más importantes de la ERIgG4 son: 1) infiltrados linfoplasmocíticos densos; 2) fibrosis tipo esteliforme o en un patrón irregular en forma de espiral, parecido a una estera de paja; y 3) flebitis obliterante. Algunas veces se observan la formación de centros germinales y la infiltración por eosinófilos en los tejidos afectados ${ }^{50}$. La inmunohistoquímica para lgG4 muestra una infiltración rica de células plasmáticas; un número mayor de 50 células con tinción positiva a IgG4 bajo un campo de alto poder se considera altamente específico, pero se han propuesto algunos otros puntos de corte ${ }^{50}$.En etapas más avanzadas de la enfermedad, la inflamación puede haber pasado y predominar la fibrosis; en estos casos hay que hacer tinción para células plasmáticas ricas en IgG. La razón $\lg 4 / \lg G$ debe ser mayor de 0.4 y es obligatoria para el diagnóstico de ER-IgG450.

\section{Manifestaciones urológicas}

\section{Pseudotumor renal}

Es una lesión que mimetiza a un carcinoma renal y resulta una forma de presentación de la enfermedad sumamente inusual, con menos de cinco casos reportados en la literatura hasta el día de hoy ${ }^{51}$. Es difícil diferenciar entre un pseudotumor renal secundario a ER-IgG4 y una lesión maligna basándonos solo en los estudios de imagen ${ }^{52}$. El ultrasonido renal puede mostrar lesiones hipoecogénicas o edema del parénquima renal, y la tomografía con medio de contraste intravenoso puede evidenciar lesiones de baja densidad, únicas o múltiples ${ }^{53}$.

La resonancia magnética puede mostrar tumores renales isointensos o hipointensos en T1, e imágenes hipointensas en T2, y mostrar realce homogéneo tras la administración de medio de contraste ${ }^{53}$. El diagnóstico diferencial definitivo lo dará una biopsia, y el tratamiento será mediante corticoesteroides, con los cuales la lesión mejorará significativamente, aumentando la función renal y disminuyendo la concentración de IgG4 circulante ${ }^{51}$.

\section{Pseudotumores de la pelvis renal y del uréter}

La ER-IgG4 puede manifestarse como una lesión única en la pelvis renal o en el uréter, causando defectos de llenado y mimetizando a un carcinoma urotelial. Solo existen cuatro casos reportados en la literatura ${ }^{54-57}$. La mayoría de los casos que han reportado lesiones renales o ureterales se acompañan de lesiones sincrónicas o metacrónicas en otros órganos ${ }^{56}$. El análisis general de orina en estos pacientes suele 
mostrar anormalidades moderadas. La manifestación de estas lesiones en los estudios de imagen es igual que la de los pseudotumores renales, pero en caso de que el pseudotumor cause obstrucción de la vía urinaria existirán cólico renoureteral e hidronefrosis.

Dentro de los hallazgos tomográficos reportados para los pseudotumores de la pelvis renal y el uréter se encuentran hidronefrosis, tumor en la unión ureteropiélica y tumor ureteral. En la resonancia magnética se han reportado lesiones isointensas en T1 e hipointensas en T2. La PET ha mostrado un metabolismo de la glucosa elevado ${ }^{54,55,57}$. El manejo de estas lesiones es con esteroides, aunque por su similitud con un carcinoma urotelial es frecuente que se realice nefroureterectomía con rodete vesical, realizando el diagnóstico de ER-IgG4 al estudiar la pieza quirúrgica ${ }^{54,57}$.

Existe un caso de pseudotumor ureteral reportado en la literatura ${ }^{58}$ que se manifestó con hidronefrosis, engrosamiento de la pared ureteral y deterioro de la función renal. Se realizó nefroureterectomía y el diagnóstico final fue dado por el análisis de la pieza patológica. Se dio manejo posterior con esteroides y se logró una mejoría importante de la función renal.

\section{Fibrosis retroperitoneal}

La FRP puede manifestarse clínicamente con dolor vago abdominal y en la espalda baja, acompañado de síntomas inespecíficos como fiebre, mialgia, anorexia y pérdida de peso. Sin embargo, la mitad de los pacientes permanecen asintomáticos, y conforme avanza la enfermedad esta puede causar compresión extrínseca del uréter llevando a pérdida de la función renal, así como a hidronefrosis; la obstrucción ureteral se encuentra en el $60-80 \%$ de los pacientes con FRT secundaria a ER-IgG4 ${ }^{59,60}$.

El diagnostico de FRP se basa en los hallazgos radiológicos y en la biopsia. Las concentraciones séricas de IgG4 pueden o no estar elevadas. Un ultrasonido renal puede dar información valiosa sobre el grado de obstrucción en el uréter y los riñones. La tomografía computada y la resonancia magnética pueden aportar información útil sobre la extensión de la enfermedad.

Algunos autores han propuesto clasificar la FRP en la ER-IgG4 como: 1) FRP; 2) aneurisma inflamatorio de la aorta abdominal; 3) una combinación de FRP y aneurisma; y 4) aortitis torácica ${ }^{61-63}$.

El manejo de la FRP incluye un alivio urgente de la obstrucción por medio de nefrostomía o de un catéter ureteral. Los pacientes con una obstrucción leve de la vía urinaria pueden ser vigilados de manera estrecha mientras se les da terapia con esteroides. El manejo definitivo de esta enfermedad es con el empleo de esteroides ${ }^{62,63}$.

\section{Pseudotumores vesicales}

Solo existen tres casos documentados de pseudotumor vesical en la literatura, y en uno de ellos se reportó un pseudotumor inflamatorio de la vejiga relacionado con ER-IgG4. Este se manifestó con hematuria macroscópica asintomática, anemia e IgG sérica elevada. En el análisis de orina se encontraron bacteriuria, hematuria, fecaluria y proteinuria. La tomografía evidencio un tumor dependiente de la pared lateral de la vejiga con adhesión al colon. La paciente se sometió a una cistectomía parcial con resección de la parte afectada del colon, encontrando en la histopatología ER-IgG4. El tratamiento definitivo fue con terapia a base de esteroides ${ }^{64-66}$.

\section{Localización de la ER-IgG4 en el uraco}

Existe un caso reportado en la literatura de afección del uraco por ER-IgG4, la cual mimetizo a un adenocarcinoma del uraco, recibiendo manejo mediante cistectomía parcial. El reporte histopatológico confirmo el diagnóstico de ER-IgG4 al mostrar una infiltración densa linfoplasmocítica con células positivas para $\operatorname{lgG} 4$, fibrosis y flebitis obliterante. Los valores séricos de $\lg G 4$ fueron de $227 \mathrm{mg} / \mathrm{dl}^{67}$.

\section{Pseudotumor de uretra}

En el año 2012 se reportó el primer caso de ERIgG4 con presentación en la uretra, el cual se encontró mediante tomografía en una paciente con dolor abdominal agudo. Se tomó biopsia de la lesión y se evidenció la ER-IgG4. Dentro de los antecedentes de la paciente, tenía diagnóstico de pancreatitis autoinmunitaria 17 años antes. Se dio tratamiento con esteroides y se logró una mejoría importante del cuadro ${ }^{68}$.

Otro estudio reportó una prevalencia de ER-IgG4 del $11 \%$ en pacientes con diagnóstico de pólipo uretral $^{69}$.

\section{Orquiepididimitis relacionada con ER-IgG4}

Se han reportado casos de orquiepididimitis bilateral, acompañada de afección de las glándulas salivales, 
con aumento de volumen del testículo izquierdo. Se realizó orquiectomía radical por la sospecha de infección por micobacterias, encontrando ER-IgG4 durante el estudio anatomopatológico. La IgG4 sérica era de $505 \mathrm{mg} / \mathrm{dl}$, con tinción positiva de testículo y epidídimo para IgG4, y con una razón lgG4/lgG mayor del $85 \%{ }^{70}$.

Otro autor ${ }^{71}$ reportó un paciente con orquitis complicada con absceso testicular, la cual fue tratada mediante orquiectomía radical, con el diagnóstico patológico confirmatorio de ER-IgG4.

\section{Pseudotumores paratesticulares}

Se han reportado algunos casos de pseudotumores paratesticulares relacionados con ER-IgG4 en pacientes de 19 a 52 años de edad. En el ultrasonido, la apariencia es la misma que la de un tumor paratesticular, y el diagnóstico viene sugerido por los antecedentes del paciente y la respuesta al tratamiento con esteroides, o por el resultado de la pieza quirúrgica después del tratamiento definitivo $0^{72-74}$.

\section{Prostatitis relacionada con ER-IgG4}

Se han reportado numerosos casos de prostatitis asociada a ER-IgG4, y en la mayoría de ellos esta se presenta acompañada de síntomas urinarios bajos,

Tabla 1. Afectación de órganos del tracto urogenital y número de casos reportados

\begin{tabular}{lc}
\hline Órgano afectado & $\mathbf{N}$ \\
\hline Riñón & 76 \\
Retroperitoneo & 19 \\
Uréter & 7 \\
Vejiga & 3 \\
Uraco & 1 \\
Testículo/epidídimo & 2 \\
Región paratesticular & 6 \\
Próstata & 22 \\
Uretra & 5 \\
\hline
\end{tabular}

en pacientes en un rango de edad de 55-73 años ${ }^{75}$. También se ha reportado que estos pacientes suelen tener unas concentraciones séricas aumentadas de lgG4 (499-1550 mg/dll) ${ }^{75-77}$ y una infiltración linfoplasmática importante de la próstata. El diagnóstico definitivo es mediante biopsia de próstata.

Es importante para el urólogo conocer esta etiología para evitar dar tratamientos innecesarios a los pacientes. Se ha visto una excelente respuesta posterior al tratamiento con esteroides, aunque algunos pacientes han mostrado una gran mejoría de los síntomas después de la resección transuretral de próstata ${ }^{75-81}$.

\section{Nefritis tubulointersticial}

La manifestación clínica de la lesión renal puede ser variable, ya sea con aparición de pseudotumores, lesión renal aguda o crónica, o proteinuria asociada a enfermedad glomerular. Las causas de la nefritis tubulointersticial se determinan mediante biopsia junto con los antecedentes clínicos del paciente, la exploración física, los marcadores séricos y los estudios de imagen. La nefritis tubulointersticial secundaria a ERIgG4 se reporta como una nefritis tubulointersticial autoinmunitaria. Es la manifestación renal más frecuente de la ER-IgG4. El $85 \%$ de los pacientes presentan anormalidades radiográficas, en el $81 \%$ con concentraciones séricas de IgG o IgG4 elevadas, y un $28 \%$ con eosinofilia. Es más común encontrar lesión renal aguda o crónica que la aparición de pseudotumores. El 84\% de estos pacientes presentan depósitos de complejos autoinmunitarios en la membrana basal tubular, donde también existe un patrón granular para IgG, C3 y las cadenas ligeras kappa y lambda.

En la tabla 1 se resumen todas las manifestaciones urológicas y el número de casos que han sido reportados hasta la fecha. Los criterios diagnósticos para la ER-IgG4 se enlistan en la tabla $2^{82,83}$.

Tabla 2. Criterios diagnósticos para la nefritis tubulointersticial por enfermedad relacionada con IgG4

\begin{tabular}{ll}
\hline Histología & $\begin{array}{l}\text { Nefritis tubulointersticial rica en células plasmáticas con más de 10 IgG4+por campo de alto poder* } \\
\text { Depósito de complejos inmunitarios en la membrana basal tubular por inmunohistoquímica o microscopía } \\
\text { electrónica }\end{array}$ \\
Imagen & $\begin{array}{l}\text { Nódulos corticales periféricos pequeños, lesiones redondas o en forma de cuña, o afectación difusa e } \\
\text { irregular }\end{array}$ \\
Serología & Elevación de IgG4 o de IgG total, hipergammaglobulinemia, eosinofilia \\
Afectación de otros órganos & $\begin{array}{l}\text { Pancreatitis autoinmunitaria, colangitis esclerosante, masas inflamatorias en cualquier órgano, aneurisma } \\
\text { aórtico, afectación de pulmón, fibrosis retroperitoneal }\end{array}$
\end{tabular}




\section{Tratamiento}

Recientemente se realizó una guía de manejo para pacientes con ER-IgG4, pero aun así no existe un tratamiento definitivo y varía de acuerdo con cada paciente. Dentro de las estrategias que pueden utilizarse están:

- Estrategia de observa y espera: es de elección en pacientes asintomáticos. En caso de afectación del riñón, el uréter o la vejiga, si se decide adoptar esta estrategia es necesario monitorizar frecuentemente la función renal y realizar ultrasonidos seriados para valorar la morfología del tracto urinario ${ }^{22,84}$.

- Glucocorticoides: la respuesta a los esteroides a dosis bajas es distintiva de la ER-IgG4, aunque resulta de interés que hasta el $30 \%$ de los pacientes con ER-IgG4 tienen resolución espontánea y un porcentaje similar es refractario o presenta recaídas.

- Otras terapias: para aquellos pacientes en quienes la terapia con esteroides no funciona o se produce una recaída, la terapia depletora de células $B$ con rituximab generalmente es adecuada. Podemos utilizar también metotrexato, ácido micofenólico y azatioprina. Hay evidencia de que con el tratamiento se modifica el curso natural de la enfermedad, tanto para normalizar la función orgánica como para evitar la fibrosis ${ }^{85-87}$.

\section{Conclusión}

Las manifestaciones urológicas de la ER-IgG4 son muchas y pueden afectar cualquier órgano del tracto genitourinario. Es importante que el urólogo tenga conocimiento de esta enfermedad, así como de sus manifestaciones clínicas, sus criterios diagnósticos y su tratamiento, para poder ofrecer una mejor atención a los pacientes.

\section{Responsabilidades éticas}

Protección de personas y animales. Los autores declaran que para esta investigación no se han realizado experimentos en seres humanos ni en animales.

Confidencialidad de los datos. Los autores declaran que han seguido los protocolos de su centro de trabajo sobre la publicación de datos de pacientes.

Derecho a la privacidad y consentimiento informado. Los autores declaran que en este artículo no aparecen datos de pacientes.

\section{Financiamiento}

Los autores declaran que no existe financiamiento.

\section{Conflicto de intereses}

Los autores declaran que no existen conflictos de intereses.

\section{Bibliografía}

1. Kamisawa T, Funata N, Hayashi $Y$, et al. A new clinicopathological entity of IgG4- related autoimmune disease. J Gastroenterol. 2003;38:982-4

2. Masaki $Y$, Dong $L$, Kurose $N$, et al. Proposal for a new clinical entity, IgG4-positive multiorgan lymphoproliferative syndrome: analysis of 64 cases of IgG4-related disorders. Ann Rheum Dis. 2009:68:1310-5.

3. Kamisawa T, Shimosegawa T, Okazaki K, et al. Standard steroid treatment for autoinmune pancreatitis. Gut. 2009;58:1504-7.

4. Hart PA, Kamisawa T, Brugge WR, et al. Long-term outcomes of autoimmune pancreatitis: a multicentre, international analysis. Gut. 2013;62:1771-6.

5. Inoue D, Yoshida K, Yoneda N, et al. IgG4-related disease: dataset of 235 consecutive patients. Medicine (Balt). 2015;94:e680.

6. Wallace ZS, Deshpande V, Mattoo H, et al. IgG4-related disease: clinical and laboratory features in one hundred twenty-five patients. Arthritis Rheum (Munch). 2015;67:2466-75.

7. Kawano M, Saeki T, Nakashima $\mathrm{H}$, et al. Proposal for diagnostic criteria for IgG4-related kidney disease. Clin Exp Nephrol. 2011;15:615-26.

8. Stone JH, Khosroshahi A, Deshpande V, et al. Recommendations for the nomenclature of IgG4-related disease and its individual organ system manifestations. Arthritis Rheum. 2012;64:3061-7.

9. Raissian Y, Nasr SH, Larsen CP, et al. Diagnosis of IgG4-related tubulointerstitial nephritis. J Am Soc Nephrol. 2011;22:1343-52.

10. Takahashi N, Kawashima A, Fletcher JG, Chari ST. Renal involvement in patients with autoimmune pancreatitis: CT and MR imaging findings. Radiology. 2007;242:791-801.

11. Inoue D, Kawano M, Yamada K. Kidney and urinary tract lesions. En: Umehara H, Okazaki K, Stone JH, Kawa S, Kawano M, editores. IgG4-related disease. Tokyo: Springer Japan; 2014. p. 99-105.

12. Kim SA, Lee SR, Huh J, et al. IgG4-associated inflammatory pseudotumor of ureter: clinicopathologic and immunohistochemical study of 3 cases. Hum Pathol. 2011;42:1178-84

13. Abe $\mathrm{H}$, Morikawa $\mathrm{T}$, Araki A, et al. IgG4-related periureteral fibrosis presenting as a unilateral ureteral mass. Pathol Res Pract. 2011; 207:712-14.

14. Kim S, Kim TG, Choi SK, et al. Immunoglobulin G4-related systemic sclerosing disease: a case involving the ureter and kidney. Korean J Urol. 2013;54:209-11.

15. Marando A, D'Ambrosio G, Catanzaro F, et al. IgG4-related disease of the ureter: report of two cases and review of the literature. Virchows Arch. 2013;462:673-8

16. Moriarty MA, Dahmoush L, Nepple KG. IgG4 related disease of the ureter (inflammatory pseudotumor). J Urol. 2014;191:1126-7.

17. Della Torre E, Mattoo H, Mahajan VS, et al. Prevalence of atopy, eosinophilia, and IgE elevation in IgG4-related disease. Allergy. 2014; 69:269-72.

18. Chen $H$, Lin W, Wang $Q$, et al. IgG4-related disease in a Chinese cohort: a prospective study. Scand J Rheumatol. 2014;43:70-4.

19. Nirula A, Glaser SM, Kalled SL, et al. What is IgG4? A review of the biology of a unique immunoglobulin subtype. Curr Opin Rheumatol. 2011;23:119-24.

20. Tanaka A, Moriyama $M, N a k a s h i m a ~ H$, et al. Th2 and regulatory immune reactions contribute to $\operatorname{lgG} 4$ production and the initiation of Mikulicz disease. Arthritis Rheum. 2012;64:254-63.

21. Tsuboi H, Matsuo N, lizuka M, et al. Analysis of IgG4 class switch-related molecules in IgG4-related disease. Arthritis Res Ther. 2012;14:R171.

22. Takeuchi M, Sato $\mathrm{Y}$, Ohno K, et al. T helper 2 and regulatory T-cell cytokine production by mast cells: a key factor in the pathogenesis of IgG4-related disease. Mod Pathol. 2014;27:1126-36.

23. Shevach EM, DiPaolo RA, Andersson J, et al. The lifestyle of naturally occurring CD4+ CD25+ Foxp3+ regulatory T cells. Immunol Rev. 2006; 212:60-73.

24. Meiler $\mathrm{F}$, Klunker $\mathrm{S}$, Zimmermann $\mathrm{M}$, et al. Distinct regulation of $\lg \mathrm{E}$, IgG4 and IgA by T regulatory cells and toll-like receptors. Allergy. 2008; 63:1455-63.

25. Satoh-Nakamura T, Kurose N, Kawanami T, et al. CD14+ follicular dendritic cells in lymphoid follicles may play a role in the pathogenesis of IgG4-related disease. Biomed Res. 2015;36:143-53. 
26. Medina F, Segundo C, Campos-Caro A, et al. The heterogeneity shown by human plasma cells from tonsil, blood, and bone marrow reveals graded stages of increasing maturity, but local profiles of adhesion molecule expression. Blood. 2002;99:2154-61.

27. Wallace ZS, Mattoo H, Carruthers M, et al. Plasmablasts as a biomarker for IgG4-related disease, independent of serum IgG4 concentrations. Ann Rheum Dis. 2015;74:190-5.

28. Mattoo H, Mahajan VS, Della-Torre E, et al. De novo oligoclonal expansions of circulating plasmablasts in active and relapsing IgG4-related disease. J Allergy Clin Immunol. 2014;134:679-87.

29. Umehara H, Okazaki K, Masaki Y, et al. A novel clinical entity, IgG4-related disease (IgG4RD): general concept and details. Mod Rheumatol/ Jpn Rheum Assoc. 2012;22:1-14.

30. Uchida K, Masamune A, Shimosegawa T, et al. Prevalence of IgG4-related disease in Japan based on nationwide survey in 2009. Int J Rheumatol. 2012;2012:358371.

31. Yamamoto M, Yajima H, Takahashi $\mathrm{H}$, et al. Everyday clinical practice in IgG4- related dacryoadenitis and/or sialadenitis: results from the SMART database. Mod Rheumatol. 2015;25:199-204.

32. Wallace ZS, Deshpande V, Mattoo H, et al. IgG4-related disease: clinical and laboratory features in 125 patients. Arthritis Rheumatol. 2015;67:2466-75.

33. Mannion M. Cron RQ. Successful treatment of pediatric IgG4 related systemic disease with mycophenolate mofetil: case report and a review of the pediatric autoimmune pancreatitis literature. Pediatr Rheumato Online J. 2011;9:1

34. Laco J, Podhola M, Kamaradova K, et al. Idiopathic vs. secondary retroperitoneal fibrosis: a clinicopathological study of 12 cases, with emphasis to possible relationship to IgG4-related disease. Virchows Arch Int $J$ Pathol. 2013:463:721-30.

35. Kalapesi FB, Garrott HM, Moldovan C, et al. IgG4 orbital inflammation in a 5-year old child presenting as an orbital mass. Orbit. 2013;32:137-40.

36. Umehara H, Okazaki K, Masaki Y, et al. Comprehensive diagnostic criteria for IgG4-related disease (IgG4-RD), 2011. Mod Rheumatol. 2012; 22:21-30.

37. Kawano M, Saeki T, Nakashima $H$, et al. Proposal for diagnostic criteria for IgG4- related kidney disease. Clin Exp Nephrol. 2011;15:615-26.

38. Shimosegawa T, Chari ST, Frulloni L, et al. International consensus diagnostic criteria for autoimmune pancreatitis: guidelines of the International Association of Pancreatology. Pancreas. 2011;40:352-8.

39. Della Torre E, Mattoo H, Mahajan VS, et al. Prevalence of atopy, eosinophilia, and IgE elevation in IgG4-related disease. Allergy. 2014; 69:269-72.

40. Chen H, Lin W, Wang Q, et al. IgG4-related disease in a Chinese cohort: a prospective study. Scand J Rheumatol. 2014;43:70-4.

41. Yamamoto M, Takahashi H, Shinomura Y. Mechanisms and assessment of IgG4-related disease: lessons for the rheumatologist. Nat Rev Rheumatol. 2014;10:148-59.

42. Cheuk W, Yuen HK, Chan AC, et al. Ocular adnexal lymphoma associated with IgG4+ chronic sclerosing dacryoadenitis: a previously undescribed complication of IgG4-related sclerosing disease. Am J Surg Pathol. 2008;32:1159-67.

43. Venkataraman G, Rizzo KA, Chavez JJ, et al. Marginal zone lymphomas involving meningeal dura: possible link to IgG4-related diseases. Mod Pathol. 2011;24:355-66.

44. Takahashi H, Yamamoto M, Tabeya T, et al. The immunobiology and clinical characteristics of IgG4 related diseases. J Autoimmun. 2012;39:93-6.

45. Kiyama K, Yoshifuji H, Kandou T. Screening for IgG4-type anti-nuclear antibodies in IgG4-related disease. BMC Musculoskelet Disord. 2015;16:129.

46. Masaki Y, Kurose N, Yamamoto M, et al. Cutoff values of serum IgG4 and histopathological IgG4+ plasma cells for diagnosis of patients with IgG4-related disease. Int J Rheumatol. 2012;2012:580814.

47. Carruthers MN, Khosroshahi A, Augustin T, et al. The diagnostic utility of serum IgG4 concentrations in IgG4-related disease. Ann Rheum Dis. 2015;74:14-8.

48. Katsura M, Mori H, Kunimatsu A, et al. Radiological features of IgG4-related disease in the head, neck, and brain. Neuroradiology. 2012; 54:873-82.

49. Nakatani K, Nakamoto $Y$, Togashi K. Utility of FDG PET/CT in IgG4-related systemic disease. Clin Radiol. 2012;67:297-305

50. Deshpande V, Zen Y, Chan JK, et al. Consensus statement on the pathology of IgG4-related disease. Mod Pathol. 2012;25:1181-92.

51. Cai Y, Li H-Z, Zhang Y-S. IgG4-related inflammatory pseudotumor of the kidney mimicking renal cell carcinoma: a case report. Oncology Letters. 2016;11:3438-40.

52. Saeki T. Kawano M. IgG4-related kidney disease. Kidney Int. 2014:85:251-7.

53. Kuroda N, Nao T, Fukuhara H, et al. IgG4-related renal disease: clinical and pathological characteristics. Int J Clin Exp Pathol. 2014;7:6379-85.

54. Tan $\mathrm{TJ}, \mathrm{Ng} \mathrm{YL}$, Tan $\mathrm{D}$, et al. Extrapancreatic findings of IgG4 related disease. Clin Radiol. 2014;69:209-18.

55. Wang $Y$, Chen X, Luo R, et al. IgG4-related systemic disease mimicking renal pelvic cancer: a rare case. World J Surg Oncol. 2014;12:395

56. Yoshino $\mathrm{T}$, Moriyama $\mathrm{H}$, Fukushima $\mathrm{M}$, et al. A case of IgG4-related retroperitoneal fibrosis mimicking renal pelvic cancer. Urol Int. 2012; 90:365-8.
57. Nishi S, Imai N, Yoshida K, et al. Clinicopathological findings of immunoglobulin G4-related kidney disease. Clin Exp Nephrol. 2011;15:810-9.

58. Park HG, Kim KM. IgG4-related inflammatory pseudotumor of the renal pelvis involving renal parenchyma, mimicking malignancy. Diagn Pathol. 2017:11:12.

59. Lei WH, Xin J, Shao CX, et al. IgG4 related kidney disease mimicking malignant ureter tumor: case report and literature review. Medicine (Balt). 2016:95:e2550.

60. Hara N, Kawaguchi M, Takeda K, et al. Retroperitoneal disorders associated with IgG4-related autoimmune pancreatitis. World J Gastroenterol. 2014;20:16550-8.

61. Corradi D, Maestri R, Palmisano A, et al. Idiopathic retroperitoneal fibrosis: clinicopathologic features and differential diagnosis. Kidney Int. 2007;72:742-53.

62. Vaglio A, Salvarani C, Buzio C. Retroperitoneal fibrosis. Lancet. 2006; 367:241-51.

63. Kottra JJ, Dunnick NR. Retroperitoneal fibrosis. Radiol Clin North Am. 1996;34:1259-75.

64. Zen Y, Kasashima S, Inoue D. Retroperitoneal and aortic manifestations of immunoglobulin G4-related disease. Semin Diagn Pathol. 2012·29:212-8.

65. Park S, Ro JY, Lee DH, et al. Immunoglobulin G4-associated inflammatory pseudotumor of urinary bladder: a case report. Ann Diagn Pathol. 2013;17:540-3.

66. Montironi R, Scarpelli M, Cheng L, et al. Immunoglobulin G4-related disease in genitourinary organs: an emerging fibroinflammatory entity often misdiagnosed preoperatively as cancer. Eur Urol. 2013;64:865-72.

67. Dropkin BM, Ingimarsson JP, Jones JD, et al. Immunoglobulin G4-related disease in the urinary bladder. Int J Urol. 2015;22:605-7.

68. Dum TW, Zhang D, Lee EK. IgG4-related disease in a urachal tumor. Case Rep Urol. 2014;2014:275850.

69. Choi JW, Kim SY, Moon KC, et al. Immunoglobulin G4-related sclerosing disease involving the urethra: case report. Korean J Radiol. 2012; 13:803-7.

70. Williamson SR, Scarpelli M, López-Beltran A, et al. Urethral caruncle: a lesion related to IgG4-associated sclerosing disease? J Clin Pathol. 2013;66:559-62.

71. Migita K, Miyashita T, Mizuno A, et al. IgG4-related epididymo-orchitis associated with bladder cancer: possible involvement of BAFF/BAFF-R interaction in IgG4-related urogenital disease. Mod Rheumatol. 2014; 24:188-94.

72. de Buy Wenniger LM, Scheltema JM, Verheij J, et al. Testicular inflammation as a new manifestation of IgG4-associated disease. Urology. 2013;82:e15-6.

73. Bösmüller $\mathrm{H}$, von Weyhern $\mathrm{CH}$, Adam $\mathrm{P}$, et al. Paratesticular fibrous pseudotumor — an IgG4- related disorder? Virchows Arch. 2011;458:109-13.

74. Karashima T, Taniguchi $Y$, Shimamoto $T$, et al. IgG4-related disease of the paratestis in a patient with Wells syndrome: a case report. Diagn Pathol. 2014:9:225

75. Hart PA, Moyer AM, Yi ES, et al. IgG4-related paratesticular pseudotumor in a patient with autoimmune pancreatitis and retroperitoneal fibrosis: an extrapancreatic manifestation of IgG4-related disease. Hum Pathol. 2012;43:2084-7.

76. Yoshimura Y, Takeda S, leki Y, et al. IgG4-associated prostatitis complicating autoimmune pancreatitis. Intern Med. 2006:45:897-901.

77. Uehara $\mathrm{T}$, Hamano $\mathrm{H}$, Kawakami $\mathrm{M}$, et al. Autoimmune pancreatitis associated prostatitis: distinct clinicopathological entity. Pathol Int. 2008;58:118-25

78. Buijs J, de Buy Maillette, Wenniger L, et al. Immunoglobulin G4-related prostatitis: a case-control study focusing on clinical and pathologic characteristics. Urology. 2014;83:521-6.

79. Hart PA, Smyrk TC, Chari ST. IgG4-related prostatitis: a rare cause of steroid-responsive obstructive urinary symptoms. Int J Urol. 2013; 20:132-4.

80. Nishimori I, Kohsaki T, Onishi S, et al. IgG4-related autoimmune prostatitis: two cases with or without autoimmune pancreatitis. Intern Med. 2007;46:1983-9

81. Bourlon MT, Sánchez-Ávila M, Chablé-Montero F, et al. IgG4-related autoimmune prostatitis: is it an unusual or underdiagnosed manifestation of IgG4-related disease? Case Rep Urol. 2013;2013:295472.

82. Nakai $Y$, Kagebayashi $Y$, Matsumoto $Y$, et al. IgG4-related prostatitis associated with retroperitoneal fibrosis: a case report. Hinyokika Kiyo. 2013:59:781-4.

83. Saeki T, Nishi S, Imai N, et al. Clinicopathological characteristics of patients with IgG4-related tubulointerstitial nephritis. Kidney Int. 2010;78:1016-23.

84. Raissian Y, Nasr SH, Larsen CP, et al. Diagnosis of IgG4-related tubulointerstitial nephritis. J Am Soc Nephrol. 2011;22:1343-52.

85. Khosroshahi A, Wallace ZS, Crowe JL, et al. International consensus guidance statement on the management and treatment of IgG4-related disease. Arthritis Rheumatol. 2015;67:1688-99.

86. Khosroshahi A, Carruthers MN, Deshpande V, et al. Stone rituximab for the treatment of IgG4-related disease: lessons from 10 consecutive patients. Medicine (Balt). 2012;91:57-66.

87. Vasaitis L. IgG4-related disease: a relatively new concept for clinicians. Eur J Intern Med. 2016;27:1-9. 